\title{
Generalized and subset integrated autoregressive bilinear time series models with applications
}

\author{
Johnson Funminiyi OJO \\ University of Ibadan, Nigeria, E-mail: jfunminiyiojo@yahoo.co.uk \\ Phone: +2348033810739
}

\begin{abstract}
Generalized integrated autoregressive bilinear model which is capable of achieving stationary for all non linear series is proposed and compared with subset generalized integrated autoregressive bilinear model using the residual variance to see which perform better. The parameters of the proposed models are estimated using Newton-Raphson iterative method and Marquardt algorithm and the statistical properties of the derived estimates were investigated. An algorithm was proposed to eliminate redundant parameters from the full order generalized integrated autoregressive bilinear models. To determine the order of the models, Akaike Information Criterion (AIC) and Bayesian Information Criterion (BIC) were adopted. Generalized integrated autoregressive bilinear models are fitted to Wolfer sunspot numbers and stationary conditions are satisfied. Generalized integrated autoregressive bilinear model performed better than subset generalized integrated autoregressive bilinear model.
\end{abstract}

Keywords: Stationary, Newton-Raphson, Residual Variance, Marquardt Algorithm and Parameters.

\section{INTRODUCTION}

The bilinear time series models have attracted considerable attention during the last years. An overview of models and their application can be found in Granger and Anderson (1978), Subba Rao (1981), Pham and Tran (1981), Gabr and Subba Rao (1981), Rao et al., (1983), Liu (1992), Cathy (1997), Gonclaves et al., (2000), Shangodoyin and Ojo (2003), Wang and Wei (2004), Boonchai and Eivind (2005), Bibi (2006), Doukhan et al., (2006), Drost et al., (2007), Usoro and Omekara (2008) and Ojo (2009). The bilinear modes studied by the above researchers could not achieve stationarity for all nonlinear series. One-dimensional bilinear time series model that could achieve stationary for all non linear series was developed, for details see Shangodyin et al., (2010) and Ojo (2010). In this paper we proposed generalized integrated autoregressive and subset bilinear models that could achieve stationary for all non linear real series.

\section{MATERIALS AND METHODS}

Proposed generalized bilinear time series models: We define generalized bilinear (GBL) time series models as follows:

Model 1(M1)
$\psi(B) X_{t}=\phi(B) \nabla^{d} X_{t}+\sum_{k=1}^{r} \sum_{l=1}^{s} b_{k l} X_{t-k} e_{t-l}+e_{t}$,

denoted as GBL (p, d, 0, r, s)

where $\phi(B)=1-\phi_{1} B-\phi_{2} B^{2} \ldots \ldots-\phi_{p} B^{p}$ and

$X_{t}=\psi_{1} X_{t-1}+\ldots . .+\psi_{p+d} X_{t-p-d}+b_{11} X_{t-1} e_{t-1}+\ldots \ldots+b_{r s} X_{t-r} e_{t-s}+e_{t}$

$\phi_{1}, \ldots, \phi_{p}$ are the parameters of the autoregressive component; $b_{11}, \ldots \ldots . . ., b_{r s}$ are the parameters of the nonlinear component and $\phi(B)$ is the autoregressive operator. $p$ is the order of the autoregressive component; $q$ is the order of the moving average process; $r, s$ is the order of the nonlinear component and $\psi(B)=\nabla^{d} \phi(B)$ is the generalized autoregressive operator; $\nabla^{d}$ is the differencing operator and $d$ is the degree of consecutive differencing required to achieve stationary.

Model 2 (M 2)

$$
X_{t}=\sum_{i=1}^{l} \psi_{p_{i}+d} X_{t-p_{i}-d}+\sum_{j=1}^{m} b_{r_{j} s_{j}} X_{t-r_{j}} e_{t-s_{j}}+e_{t}
$$

denoted as GSBL (p, d, o, r, s). 
where, $p_{i}$ is the order of subset autoregressive component and $r_{j} s_{j}$ is the order of subset nonlinear component. In the models above, $e_{t}$ are independently and identically distributed as $N\left(0, \sigma_{e}^{2}\right)$ and the models are assume to be invertible (see section 2.2).

Stationary and Convergence of Generalized Bilinear Models: In this section, we give a sufficient condition for the existence of strictly stationary process and convergence conforming to the bilinear model (2.1). This we do through the following theorem.

\section{Theorem}

Let $\left\{e_{t}, t \in Z\right\}$ be a sequence of independent identically distributed random variables defined on a probability space $(\Omega, F, P)$ such that $\mathrm{E} \mathrm{e}_{\mathrm{t}}=0$ and $E e_{t}^{2}=\sigma^{2}<\infty$.Let $\Psi, \mathrm{B}_{1}, \mathrm{~B}_{2}, \ldots, \mathrm{B}_{\mathrm{q}}$ be $\mathrm{q}+1$ matrices each of order $\mathrm{p} \times \mathrm{p}$ and

$$
\begin{aligned}
\Gamma_{1}=\Psi \otimes & \Psi+\sigma^{2}\left(B_{1} \otimes B_{1}\right), \\
\Gamma_{j}=\sigma^{2}\left[B_{j} \otimes\right. & \left(\Psi^{j-1} B_{1}+\Psi^{j-2} B_{2}+\ldots . .+\Psi B_{j-1}\right) \\
& +\left(\Psi^{j-1} B_{1}+\Psi^{j-2} B_{2}+\ldots+\Psi B_{j-1}\right) \otimes B_{j} \\
& \left.+\left(B_{j} \otimes B_{j}\right)\right], \quad j=2,3, \ldots \ldots . .5 .
\end{aligned}
$$

Suppose all the eigenvalues of the matrix

$$
\underset{p^{2} q \times p^{2} q}{L}=\left(\begin{array}{ccccc}
\Gamma_{1} & \Gamma_{2} & \ldots \ldots & \Gamma_{q-1} & \Gamma_{q} \\
I_{p^{2}} & 0 & \ldots \ldots . . & 0 & 0 \\
0 & I_{p^{2}} & & 0 & 0 \\
0 & 0 & & I_{p^{2}} & 0
\end{array}\right)
$$

have moduli less than unity, i.e, $\rho(L)=\lambda<1$. Let $\underset{p \times 1}{C}$ be a given column vector. Then there exists a vector valued strictly stationary process $\left\{X_{t}, t \in Z\right\}$ conforming to the vector form of generalized

bilinear

model $\mathbf{X}_{t}=\Psi \mathbf{X}_{t-1}+\sum_{j=1}^{s} \mathbf{B}_{j} \mathbf{X}_{t-j} e_{t-j}+\mathbf{C} e_{t}$ for every $\mathrm{t}$ in $\mathrm{Z}$.

\section{Proof}

The proof of the theorem for the sake of simplicity is carried out in the following steps.

\section{Step 1}

Let the process $\left\{\mathbf{S}_{n, t}, n, t \in Z\right\}$ be defined as follows:

$$
S_{n, t}=C e+\left(\Psi+B e_{t-1}\right) S_{n-t, t-1}+B_{2} S_{n-2, t-2} e_{t-2}+\ldots+B_{s} S_{n-s, t-s} e_{t-s} \text {, }
$$

if $\mathrm{n}>0$ for every $\mathrm{t}$ in $\mathrm{Z}$.

We show that $\lim _{n \rightarrow \infty} \mathbf{S}_{n, t}$ exists almost surely for every $\mathrm{t}$ in $\mathbf{Z}$. If $\mathbf{X}_{t}$ is the almost sure limit of $\left\{\mathbf{S}_{n, t}, n \geq 1\right\}$ for every $\mathrm{t}$ in $\mathrm{Z}$, then it is obvious that the process $\left\{\mathbf{X}_{t}, t \in Z\right\}$ conforms to the bilinear model (2.1). It is also easy to check that for every fixed $n$ in $Z,\left\{\mathbf{S}_{n, t}, t \in Z\right\}$ is a strictly stationary process.

\section{Step 2}

Let $\quad \mathbf{S}_{n, t}=\mathbf{S}_{n, t}-\mathbf{S}_{n-1, t} t \in Z$. We show that $E\left|\left(\mathbf{s}_{n, t}\right)_{i}\right| \leq K \lambda^{n / 2}$ for every $n \geq 0$ and $\mathrm{i}=1,2, \ldots, \mathrm{p}$, where $\mathrm{K}$ is a positive constant. Since $\lambda<1$, this then implies that $\left\{\mathbf{S}_{n, t}, n \geq 1\right\}$ converges almost surely for every $\mathrm{t}$ in $\mathrm{Z}$. (If $\left\{a_{n,} n \geq 1\right\}$ is $a$ sequence of real numbers satisfying $\left|a_{n}-a_{n-1}\right| \leq K \lambda^{n}$ for every $n \geq 2$ for some positive constant $\mathrm{K}$ and $\lambda<1$, then it is easy to show that $\left\{a_{n,} n \geq 1\right\}$ is a Cauchy sequence of real numbers.)

\section{Step 3}

First, we settle the question of integrability of the $\mathbf{S}_{n, t}$ 's. Note that

$$
\begin{aligned}
& \mathbf{s}_{n, t}= \mathbf{S}_{n, t}-\mathbf{S}_{n-1, t} \\
&= \\
&\left(\Psi+B_{1} e_{t-1}\right) \mathbf{s}_{n-1, t-1}+B_{2} \mathbf{s}_{n-2, t-2} e_{t-2}+\ldots . .+B_{s} \mathbf{s}_{n-s, t-s} e_{t-s} \\
&=Q_{n}\left(e_{t-1}, e_{t-2}, \ldots . ., e_{t-n}\right) \mathbf{s}_{0, t-n} \\
&=Q_{n}\left(e_{t-1}, e_{t-2}, \ldots . ., e_{t-n}\right) C e_{t-n},
\end{aligned}
$$


where $Q_{n}\left(e_{t-1}, e_{t-2}, \ldots . ., e_{t-n}\right)$ is a matrix of order $\mathrm{p} \times \mathrm{p}$ and each entry of this matrix is a polynomial in $e_{t-1}, e_{t-2}, \ldots . ., e_{t-n}$ in which the power index of each $e_{t-j}$ is either 0 or 1 . Consequently, every entry in $Q_{n}\left(e_{t-1}, e_{t-2}, \ldots . ., e_{t-n}\right)$ and hence in $\mathbf{s}_{n, t}$ is integrable. It is clear that distribution of $\mathbf{s}_{n, t}$ does not depend on $\mathrm{t}$.

\section{Step 4}

It is convenient to deal with the following processes. Define

$\mathbf{s}_{n, t}^{*}=Q_{n}\left(e_{t-1}, e_{t-2}, \ldots . ., e_{t-n}\right) C$, if $\mathrm{n}>0$ for every $\mathrm{t}$ in Z. Equivalently, $\mathbf{s}_{n, t}=\mathbf{s}_{n, t}^{*} e_{t-n}, \mathrm{n}, t \in Z$.

From the remark made regarding the $Q_{n}(.)^{\prime} s$ in step 3 , it is obvious that every entry in $\mathbf{s}_{n, t}^{*}$ is square integrable. Further, it is easy to check that $\mathbf{s}_{n, t}^{*} s$ satisfy the following equation.

$\mathbf{s}_{n, t}^{*}=\left(\Psi+B_{1} e_{t-1}\right) \mathbf{s}_{n-1, t-1}^{*}+B_{2} \mathbf{s}_{n-2, t-2}^{*} e_{t-2}+\ldots .+B_{s} \mathbf{s}_{n-s, t-s}^{*} e_{t-s}$

for every $\mathrm{n}, \mathrm{t}$ in Z. Also, the distribution of $\mathbf{s}_{n, t}^{*}$ does not depend on $\mathrm{t}$, since the $e_{t} s$ are independently identically distributed. Since $\mathbf{s}_{n, t}=\mathbf{s}_{n, t}^{*} e_{t-n}$ for all $\mathrm{n}$ and $\mathrm{t}$ in $\mathrm{Z}$.

$$
\begin{aligned}
& E\left|\left(\mathbf{s}_{n, t}\right)_{i}\right|=E\left|\left(\mathbf{s}_{n, t}^{*}\right)_{i}\right|\left|e_{t-n}\right| \\
& \leq\left(E\left(\left(\mathbf{s}_{n, t}^{*}\right)_{i}\right)^{2}\right)^{1 / 2}\left(E e_{t-n}^{2}\right)^{1 / 2} \leq \sigma\left(E\left(\left(\mathbf{s}_{n, t}^{*}\right)_{i}\right)^{2}\right)^{1 / 2}
\end{aligned}
$$

for every $i=1,2, \ldots \ldots, p$. It suffices to obtain an upper bound for $E\left(\left(\mathbf{s}_{n, t}^{*}\right)_{i}\right)^{2}$ for every $\mathrm{i}=1,2, \ldots, \mathrm{p}$ and $\mathrm{n}, \mathrm{t}$ in

Z. For this we evaluate $E\left(\mathbf{s}_{n, t}^{*} \otimes \mathbf{s}_{n, t}^{*}\right)=M_{n}$, say

\section{Step 5}

$$
\begin{aligned}
& \text { Let } \quad D_{1}=\left(\Psi+B_{1} e_{t-1}\right) \mathbf{s}_{n-1, t-1}^{*} \quad \text { for } \quad \mathrm{i}=2,3, \ldots, \mathrm{s} . \\
& D_{i}=B_{i} \mathbf{s}_{n-1, t-1}^{*} e_{t-i} \quad \mathbf{s}_{n, t}^{*} \otimes \mathbf{s}_{n, t}^{*}=\left(\sum_{i=1}^{s} D_{i}\right) \otimes\left(\sum_{i=1}^{s} D_{i}\right)=\left\{D_{1} \otimes D_{1}\right\}+\left\{D_{1} \otimes D_{2}+D_{2} \otimes D_{2}+D_{2} \otimes D_{1}\right\}
\end{aligned}
$$

$$
\begin{aligned}
& +\left\{D_{1} \otimes D_{3}+D_{2} \otimes D_{3}+D_{3} \otimes D_{3}+D_{3} \otimes D_{2}+D_{3} \otimes D_{1}\right\}+\ldots . . \\
& +\left\{\sum_{i=1}^{s-1} D_{i} \otimes D_{s}+D_{s} \otimes D_{s}+\sum_{i=1}^{s-1} D_{s} \otimes D_{i}\right\} .
\end{aligned}
$$

We evaluate the expectation of each expression within each set of brackets \{\} in (2.4)

\section{Step 6}

We

write

$D_{1} \otimes D_{1}=\left(\left(\Psi+B_{1} e_{t-1}\right) \otimes\left(\Psi+B_{1} e_{t-1}\right)\right)\left(\mathbf{s}_{n-1, t-1}^{*} \otimes \mathbf{s}_{n-1, t-1}^{*}\right)$.

Since $\mathbf{S}_{n-1, t-1}^{*}$ is a function of $e_{t-2}, e_{t-3}, \ldots, e_{t-n}, \mathbf{s}_{n-1, t-1}^{*} \quad$ and $e_{t-1}$ are independently distributed. So,

$E\left(D_{1} \otimes D_{1}\right)=\left(\left(\Psi \otimes \Psi+\sigma^{2}\left(B_{1} \otimes B_{1}\right)\right)=\Gamma_{1} M_{n-1}\right.$.

\section{Step 7}

Expanding $\mathbf{s}_{n-1, t-1}^{*}$, we obtain

$D_{1} \otimes D_{2}=\left(\left(\Psi+B_{1} e_{t-1}\right)\left(\Psi+B_{1} e_{t-2}\right) \otimes B_{2} e_{t-2}\right)\left(\mathbf{s}_{n-2, t-2}^{*} \otimes \mathbf{s}_{n-2, t-2}^{*}\right)$

$+\left(\left(\Psi+B_{1} e_{t-1}\right) \otimes B_{2} e_{t-2}\right)\left(B_{2} \mathbf{s}_{n-3, t-3}^{*} e_{t-3} \otimes \mathbf{s}_{n-2, t-2}^{*}\right)+\ldots+$

$\left(\left(\Psi+B_{1} e_{t-1}\right) \otimes B_{2} e_{t-2}\right)\left(B_{s} \mathbf{s}_{n-1-s, t-1-s}^{*} e_{t-1-s} \otimes \mathbf{s}_{n-2, t-2}^{*}\right)$.

Therefore, $E\left(D_{1} \otimes D_{2}\right)=\sigma^{2}\left(\left(\Psi B_{1}\right) \otimes B_{2}\right) M_{n-2}$

In a similar fashion, we can show that $E\left(D_{2} \otimes D_{1}\right)=\sigma^{2}\left(B_{2} \otimes\left(\Psi B_{1}\right)\right) M_{n-2}$

and $E\left(D_{2} \otimes D_{2}\right)=\sigma^{2}\left(B_{2} \otimes B_{2}\right) M_{n-2}$. Consequently, the expected value of the entire expression in the second set of such brackets is $\sigma^{2}\left(B_{2} \otimes\left(\Psi B_{1}\right)+\left(\Psi B_{1}\right) \otimes B_{2}+B_{2} \otimes B_{2}\right) M_{n-2}=\Gamma_{2} M_{n-2}$. 
Step 8: Pursuing ideas similar to those used in step 7 , we can show that the expected value of the entire expression in the third set of such brackets in (2.4) is

$\sigma^{2}\left(B_{3} \otimes\left(\Psi^{2} B_{1}+\Psi B_{2}\right)+\left(\Psi^{2} B_{1}+\Psi B_{2}\right) \otimes B_{3}+B_{3} \otimes B_{3}\right)=\Gamma_{3} M_{n-3}$.

Step 9: The expectations of other expressions can be evaluated analogously. Finally, we obtain

$M_{n}=E\left(\mathbf{s}_{n, t}^{*} \otimes \mathbf{s}_{n, t}^{*}\right)=\sum_{i=1}^{s} \Gamma_{i} M_{n-i}$ for all $\mathrm{n}$.

Step 10

Since $M_{n}=E\left(\mathbf{s}_{n, t}^{*} \otimes \mathbf{s}_{n, t}^{*}\right)$, we have

$$
E\left(\left(\mathbf{s}_{n, t}^{*}\right)_{i}\right)^{2} \leq K^{\prime} \lambda^{n}
$$

where $\rho(L)=\lambda<1$ and $K^{\prime}$ is a positive constant.

Description of Algorithm for Fitting Subset Generalized Bilinear Models: For the sake of simplicity, we will break the algorithm down into the following steps.

Step 1: Fit various order of autoregressive integrated model of the form

$$
X_{t}=\psi_{1} X_{t-1}+\ldots \ldots+\psi_{p+d} X_{t-p-d}+e_{t}
$$

Step 2: Choose the model for which Akaike Information Criterion (AIC) is minimum among various order fitted in step 1.

Step 3: Fit possible subsets of chosen model in step 2 using $2^{q}-1$ subsets approach Hagan and Oyetunji (1980).

Step 4: Choose the model for which AIC is minimum among the fitted models in step 3 to have the best subset model.

Step 5: Fit various order of the generalized bilinear model of the form $X_{t}=\psi_{1} X_{t-1}+\ldots \ldots .+\psi_{p+d} X_{t-p-d}+b_{11} X_{t-1} e_{t-1}+\ldots \ldots$.

$$
+b_{r s} X_{t-r} e_{t-s}+e_{t}
$$

and choose the model for which AIC is minimum

Step 6: Fit possible subsets of chosen model in step 5 using $2^{q}-1$ subsets approach Shangodoyin and Ojo (2003).
Step 7: The model with the minimum AIC is the best subset generalized bilinear model.

\section{Estimation of the Parameters of Generalized Integrated Autoregressive Bilinear}

\section{Models}

The joint density function of $\left(e_{m}, e_{m+1}, \ldots ., e_{n}\right)$ where $\mathrm{m}=\max (r, s)$ is given by

$$
\frac{1}{\left(2 \pi \sigma_{e}^{2}\right)^{(n-m+1) / 2}} \exp \left(\frac{1}{-2 \sigma_{e}^{2}} \sum_{m}^{n} e_{t}^{2}\right)
$$

Since the Jacobian of the transformation from $\left(e_{m}, e_{m+1}, \ldots ., e_{n}\right)$ to $\left(X_{m}, X_{m+1}, \ldots ., X_{n}\right)$ is unity, the likelihood function of $\left(X_{m}, X_{m+1}, \ldots, X_{n}\right)$ is the same as the joint density function of $\left(e_{m}, e_{m+1}, \ldots ., e_{n}\right)$. Maximising the likelihood function is the same as minimizing the function $Q(G)$, where

$$
Q(G)=\sum_{i=m}^{n} e_{t}^{2}
$$

with respect to the parameter $G^{\prime}=\left(\psi_{1}, \ldots ., \psi_{p} ; B_{11}, \ldots ., B_{r s}\right)$

Then the partial derivatives of $\mathrm{Q}(\mathrm{G})$ are given by

$\frac{d Q(G)}{d G_{i}}=2 \sum_{t=m}^{n} e_{t} \frac{d e_{t}}{d G_{i}} \quad(\mathrm{i}=1,2, \ldots \ldots, \mathrm{R})$

$\frac{d^{2} Q(G)}{d G_{i} d G_{j}}=2\left(\sum_{t=m}^{n} e_{t} \frac{d e_{t}}{d G_{i}} \frac{d e_{t}}{d G_{j}}+\sum_{t=m}^{n} e_{t} \frac{d^{2} e_{t}}{d G_{i} d G_{j}}\right)$

where these partial derivatives of $\mathrm{e}(\mathrm{t})$ satisfy the recursive equations

$\frac{d e_{t}}{d \psi_{i}}+\sum_{j=1}^{s} W_{t}(t) \frac{d e_{t-j}}{d \psi_{i}}=\left\{\begin{array}{c}1, \text { if } \mathrm{i}=0 \\ \mathrm{x}_{\mathrm{t}-\mathrm{i}}, \text { if } \mathrm{i}=1,2, \ldots, \mathrm{p}\end{array}\right.$ 
$\frac{d e_{t}}{d B_{k m i}}+\sum_{j=1}^{s} W_{j}(t) \frac{d e_{t-j}}{d B_{k m i}}=-X_{t-k} e_{t-m}\left(\mathrm{k}=1,2, \ldots, \mathrm{r} ; \mathrm{m}_{\mathrm{i}}\right.$ $=1,2, \ldots, \mathrm{s})$

$\frac{d^{2} e_{t}}{d \psi_{i} d \psi_{i}^{\prime}}+\sum_{j=1}^{s} W_{j}(t) \frac{d^{2} e_{t-j}}{d \psi_{i} d \psi_{i}^{\prime}}=0\left(\mathrm{i}, \mathrm{i}^{\prime}=0,1,2\right.$ $\ldots, p)$

$\frac{d^{2} e_{t}}{d \psi_{i} d B_{k m i}}+\sum_{j=1}^{s} W_{j}(t) \frac{d^{2} e_{t-j}}{d B_{k m i} d \phi_{i}}+X_{t-k} \frac{d^{2} e_{t-m i}}{d \psi_{i}}=0$

$$
\left(i=0,1,2, \ldots, p ; \quad k_{i} \quad=1,2, \ldots, r ; \quad m_{i}=1,2, \ldots, s\right)
$$

$\frac{d^{2} e_{t}}{d B_{k m l} d B_{k m i}^{\prime}}+\sum_{j=1}^{s} W_{j}(t) \frac{d^{2} e_{t-j}}{d B_{k m l} d B_{k m i}^{\prime}}+X_{t-k}^{\prime} \frac{d^{2} e_{t-m i}}{d B_{k m i}}=-X_{t-k} \frac{d e_{t-m}}{d B_{k m i}^{\prime}}$

$\left(k, k^{\prime}=1,2, \ldots, r ; m_{i} m_{i}^{\prime}=1,2, \ldots, s\right)$

$$
W_{j}(t)=\sum_{j=1}^{s} B_{i j} X_{t-j}
$$

We assume $e_{t}=0(t=1,2, \ldots, m-1)$ and also

$$
\begin{array}{r}
\frac{d e_{t}}{d G_{i}}=0, \frac{d^{2} e_{t}}{d G_{i} d G_{j}}=0,(\mathrm{i}, \mathrm{j}=1,2, \ldots, \\
\mathrm{R} ; \mathrm{t}=1,2, \ldots, \mathrm{m}-1)
\end{array}
$$

From $e_{\mathrm{t}}=0 \quad(\mathrm{t}=1,2, \quad \ldots, \mathrm{m}-1)$, $\frac{d e_{t}}{d G_{i}}=0, \frac{d^{2} e_{t}}{d G_{i} d G_{j}}=0$, and

$$
\begin{aligned}
& \frac{d e_{t}}{d B_{k m i}}+\sum_{j=1}^{s} W_{j}(t) \frac{d e_{t-j}}{d B_{k m i}}=-X_{t-k} e_{t-m} \\
&\left(\mathrm{k}=1,2, \ldots, \mathrm{r} ; \mathrm{m}_{\mathrm{i}}=1,2, \ldots, \mathrm{s}\right),
\end{aligned}
$$

it follows that the second order derivatives with respect to $\psi_{i}(i=0,1,2, \ldots, p)$ are zero. For a given set of values $\left\{\phi_{i}\right\}$ and $\left\{\mathrm{B}_{\mathrm{ij}}\right\}$ one can evaluate the first and second order derivatives using the recursive equations, 2.7, 2.8 and 2.11. Now let

$$
\mathbf{V}^{\prime}(\mathbf{G})=\frac{d Q(\mathbf{G})}{d \mathbf{G}_{1}}, \frac{d Q(\mathbf{G})}{d \mathbf{G}_{2}}, \ldots \ldots . ., \frac{d Q(\mathbf{G})}{d \mathbf{G}_{k}}
$$

and let $\mathbf{H}(\mathbf{G})=\left[d^{2} Q(\mathbf{G}) / d \mathbf{G}_{i} d \mathbf{G}_{j}\right]$ be a matrix of second partial derivatives as in Krzanowski (1998). Expanding $\mathbf{V}(\mathbf{G})$, near $G=\hat{G}$ in a Taylor series, we obtain $V(\hat{G})_{\hat{G}=G}=0=V(G)+H(G)(\hat{G}-G)$

Rewriting this equation we get $\hat{G}-G=-H^{-1}(G) V(G)$, and thus obtain an iterative equation given by $G^{(k+1)}=G^{(k)}-H^{-1}\left(G^{(k)}\right) V\left(G^{(k)}\right)$ where $G^{(k)}$ is the set of estimates obtained at the $k^{\text {th }}$ stage of iteration. The estimates obtained by the above iterative equations usually converge. For starting the iteration, we need to have good sets of initial values of the parameters. This is done by fitting the best subset of the linear part of the bilinear model.

\section{Estimation of the Parameters of Subset Generalized Integrated Autoregressive}

\section{Bilinear Model}

In the estimation procedure to be discussed in this section we assume that the sets of integers $\left\{k_{1}, k_{2}, \ldots, k_{l}\right\}$ and $\quad\left\{\left(r_{1}, s_{1}\right),\left(r_{2}, s_{2}\right), \ldots .,\left(r_{m} s_{m}\right)\right\}$ are fixed and known. Proceeding as in Subba Rao (1981), we can show that maximizing the likelihood function of $\left(X_{m 1}, X_{m 1+1}, \ldots, X_{N}\right)$ is the same as minimizing the function $Q(\theta)=\sum_{t=m 1}^{N} e_{t}^{2}$ with respect to the parameters $\left(\psi_{k_{1}}, \psi_{k_{2}}, \ldots ., \psi_{k_{l}} ; b_{r_{1} s_{1}}, \ldots . ., b_{r_{m} s_{m}}\right)$.

The partial derivatives of $Q(\theta)$ are

$$
\begin{aligned}
& G_{i}=\frac{d Q(\theta)}{d \theta_{i}}=2 \sum_{t=m_{1}}^{N} e_{t} \frac{d e_{t}}{d \theta_{i}}, \\
& h_{i j}=\frac{d^{2} Q(\theta)}{d \theta_{i} d \theta_{j}}=2 \sum_{t=m_{1}}^{N}\left(\frac{d e_{t}}{d \theta_{i}}\right)\left(\frac{d e_{t}}{d \theta_{j}}\right)+2 \sum_{t=m_{1}}^{N} e_{t} \cdot \frac{d^{2} e_{t}}{d \theta_{i} d \theta_{j}},
\end{aligned}
$$


where the partial derivatives satisfy the recursive equations

$$
\begin{aligned}
& \frac{d e_{t}}{d \psi_{k_{r}}}=X_{t-k_{r}}-\sum_{j=1}^{m} b_{r_{j} s_{j}} X_{t-r_{j}} \frac{d e_{t-s_{j}}}{\psi_{k_{r}}},(r=1,2,3, \ldots, l) \\
& \frac{d e_{t}}{d b_{r_{q} s_{q}}}=-X_{t-r_{q}} e_{t-s_{q}}-\sum_{j=1}^{m} b_{r_{j} s_{j}} X_{t-r_{j}} \frac{d e_{t-s_{j}}}{d b_{r_{q} s_{q}}},
\end{aligned}
$$

In the calculation of these partial derivatives, we set $e_{1}=e_{2}=\ldots=e_{m o}=0$ and

$\frac{d e_{1}}{d \theta_{i}}=\frac{d e_{2}}{d \theta_{i}}=\ldots \ldots=\frac{d e_{m o}}{d \theta_{i}}=0,(i=1,2, \ldots, R)$

Let $G^{T}(\theta)=\left(G_{1}, G_{2}, \ldots, G_{R}\right)$ and $H(\theta)=\left(h_{i j}\right)$.

In evaluating the second order partial derivatives we approximate

$h_{i j}=2 \sum_{t=m_{1}}^{N}\left(\frac{d e_{t}}{d \theta_{i}}\right)\left(\frac{d e_{t}}{d \theta_{i}}\right)$

as is done in Marquardt algorithm. Expanding $G(\hat{\theta})$ near $\hat{\theta}=\theta$ in a Taylor series, we obtain $0=G(\theta)+H(\theta)(\hat{\theta}-\theta)$.

Rewriting this equation , we get $(\hat{\theta}-\theta)=-H^{-1}(\theta) G(\theta)$ and thus obtain the Newton-Raphson iterative equation

$$
\begin{aligned}
& \theta^{(k+1)}=\theta^{(k)}-H^{-1}\left(\theta^{(k)}\right) G\left(\theta^{(k)}\right) \\
& \theta^{(k)}=\theta^{(k+1)}+H^{-1}\left(\theta^{(k)}\right) G\left(\theta^{(k)}\right)
\end{aligned}
$$

where $\theta^{(k)}$ is the set of estimates obtained at the $k^{\text {th }}$ stage of iteration. For starting the iteration, we need to have good sets of initial values of the parameters. This is done by fitting the best subset of the linear part of the bilinear model.

\section{RESULTS AND DISCUSSION}

\section{Results}

To present the application of the models proposed, we will use a real time series dataset, the Wolfer sunspot, available in Box et al., (1994). The scientists track solar cycles by counting sunspots - cool planet- sized areas on the Sun where intense magnetic loops poke through the star's visible surface. It was Rudolf Wolf who devised the basic formula for calculating sunspots in 1848; these sunspot counts are still continued.

As the Wolfer sunspot data set represent a nonstationary series, the bilinear models proposed in this paper may be applied. The Wolfer sunspot data set is considered at sample size of 50, 150 and 250. For the fitted model below we have used the algorithm and the estimation technique in the previous section.

\section{Fitted Model M1 and M2 at $t=50$}

M1

$$
\begin{aligned}
X_{t}= & 0.314548 X_{t-1}-0.458429 X_{t-2}-0.302114 X_{t-4} \\
& -0.220568 X_{t-5}-0.386159 X_{t-6}-0.002758 X_{t} \\
& -1 e_{t-1}-0.020647 X_{t-1} e_{t-2}-0.018189 X_{t-1} e_{t-3} \\
& +0.015317 X_{t-2} e_{t-1}+e_{t}
\end{aligned}
$$

M2

$$
\begin{aligned}
X_{t}= & 0.314548 X_{t-1}-0.458429 X_{t-2}-0.302114 X_{t-4}- \\
& 0.220568 X_{t-5}-0.386159 X_{t-6}-0.020351 X_{t-1} e_{t} \\
& -2-0.016095 X_{t-1} e_{t-3}+0.013653 X_{t-2} e_{t-1}+e_{t}
\end{aligned}
$$

\section{Fitted Model M1and M2 at $t=150$}

M1

$$
\begin{aligned}
\mathrm{X}_{\mathrm{t}}= & 0.412820 \mathrm{X}_{\mathrm{t}-1}-0.271125 \mathrm{X}_{\mathrm{t}-2}-0.270908 \mathrm{X}_{\mathrm{t}-3} \\
& -0.339150 \mathrm{X}_{\mathrm{t}-5^{-}} 0.293320 \mathrm{X}_{\mathrm{t}-7}+0.000325 \mathrm{X}_{\mathrm{t}} \\
& -1 \mathrm{e}_{\mathrm{t}-1}-0.020870 \mathrm{X}_{\mathrm{t}-1} \mathrm{e}_{\mathrm{t}-2}-0.002425 \mathrm{X}_{\mathrm{t}-1} \mathrm{e}_{\mathrm{t}-3} \\
& +0.018075 \mathrm{X}_{\mathrm{t}-2} \mathrm{e}_{\mathrm{t}-1}+0.009283 \mathrm{X}_{\mathrm{t}-2} \mathrm{e}_{\mathrm{t}-2}- \\
& 0.008691 \mathrm{X}_{\mathrm{t}-2} \mathrm{e}_{\mathrm{t}-3}-0.019234 \mathrm{X}_{\mathrm{t}-3} \mathrm{e}_{\mathrm{t}-1}- \\
& 0.007737 \mathrm{X}_{\mathrm{t}-3} \mathrm{e}_{\mathrm{t}-2}+\mathrm{e}_{\mathrm{t}}
\end{aligned}
$$

M2

$$
\begin{aligned}
X_{t}= & 0.412820 X_{t-1}-0.271125 X_{t-2}-0.270908 X_{t-3}- \\
& 0.339150 X_{t-5} 0.293320 X_{t-7}-0.021157 X_{t-1} e_{t} \\
& -2+0.018650 X_{t-2} e_{t-1}+0.009595 X_{t-2} e_{t-2}- \\
& 0.009477 X_{t-2} e_{t-3}-0.021380 X_{t-3} e_{t-1-} \\
& 0.008220 X_{t-3} e_{t-2}+e_{t}
\end{aligned}
$$

Fitted Model M1and M2 at $t=250$

M1

$$
\begin{aligned}
X_{t}=- & 0.239576 X_{t-2}-0.361665 X_{t-3}-0.238746 X_{t-4}- \\
& 0.325416 X_{t-5}-0.328627 X_{t-6}-0.209789 X_{t-7}- \\
& 0.365561 X_{t-8}+0.000633 X_{t-1} e_{t-1}-0.010392 X_{t} \\
& -{ }_{1} e_{t-2}+0.007590 X_{t-1} e_{t-3}+0.005443 X_{t-2} e_{t-1}
\end{aligned}
$$




$$
\begin{aligned}
& +0.000716 X_{t-2} e_{t-2}-0.005326 X_{t-2} e_{t-3}- \\
& 0.013130 X_{t-3} e_{t-1}+e_{t}
\end{aligned}
$$

M2

$$
\begin{aligned}
X_{t}= & -0.239576 X_{t-2}+0.361665 X_{t-3}-0.238746 X_{t-4} \\
& -0.325416 X_{t-5}-0.328627 X_{t-6}-0.209789 X_{t} \\
& -7-0.365561 X_{t-8}-0.009014 X_{t-1} e_{t-2}+ \\
& 0.006529 X_{t-1} e_{t-3}+0.004016 X_{t-2} e_{t-1}- \\
& 0.005079 X_{t-2} e_{t-3}-0.012555 X_{t-3} e_{t-1}+e_{t}
\end{aligned}
$$

The fitted models' residual variances, coefficient of determination( $R$-squared) and F-statistic are given in table 1(see Appendix).

\begin{tabular}{|c|c|c|c|c|c|c|}
\hline $\begin{array}{l}\text { Sample } \\
\text { size }\end{array}$ & \multicolumn{2}{|c|}{ Sample size of 50} & \multicolumn{2}{|c|}{$\begin{array}{l}\text { Sample size of } \\
150\end{array}$} & \multicolumn{2}{|c|}{$\begin{array}{l}\text { Sample size of } \\
250\end{array}$} \\
\hline Model & $\begin{array}{l}\text { Full } \\
\text { Bilinear }\end{array}$ & $\begin{array}{l}\text { Subset } \\
\text { Bilinear }\end{array}$ & $\begin{array}{l}\text { Full } \\
\text { Bilinear }\end{array}$ & $\begin{array}{l}\text { Subset } \\
\text { Bilinear }\end{array}$ & $\begin{array}{l}\text { Full } \\
\text { Bilinear }\end{array}$ & $\begin{array}{l}\text { Subset } \\
\text { Bilinear }\end{array}$ \\
\hline $\begin{array}{l}\text { Residual } \\
\text { Variance }\end{array}$ & 250.20 & 253.32 & 193.20 & 193.30 & 285.5 & 285.8 \\
\hline $\mathrm{R}^{2}$ & 0.58 & 0.57 & 0.61 & 0.60 & 0.55 & 0.54 \\
\hline $\begin{array}{l}\text { F } \\
\text { (Statistic) }\end{array}$ & 20.93 & 31.79 & 31.18 & 44.12 & 49.29 & 74.0 \\
\hline
\end{tabular}

Table1. Goodness of fit of generalized integrated autoregressive and subset bilinear models at sample sizes of 50,150 and 250 . Two models are compared, namely M1: GBL ( $p, d, 0, r, s), M 2$ : $\operatorname{GSBL}(p, d, 0, r, s)$. All models are significant at $p<0.001$.

\section{DISCUSSION}

From table 1 in the appendix, we could see the performance of the two models above using the residual variance attached to each model. The residual variance of full bilinear model is smaller than that of subset model. The proposed model gave us the best model at full model which is an improvement. The usual convention is that the subset model is always better than the full model. But in this proposed model, testing all subsets of the models to have the best model is not necessary.

\section{CONCLUSION}

This study focused on generalized integrated bilinear models that could handle all non-linear series. Generalized bilinear models at different levels of sample sizes were considered using the non-linear real series. Generalized integrated bilinear model emerged as the better model when compared with subset model. And this is an improvement in the model proposed. Moreover, estimation of parameters witnessed a unique, consistent and convergent estimator that has prevented the models from exploding, thereby making stationary possible.

\section{REFERENCES}

Bibi A. (2006) Evolutionary transfer functions of bilinear process with time varying coefficients. Computer and Mathematics with Applications 52, 3-4, 331-338.

Box, G.E.P, Jenkins, G.M and Reinsel, G.C (1994) Time series analysis; forecasting and control. 3rd Edition. Prentice Hall, Inc.

Boonaick K. Stensholt B.K. and Stensholt E. (2005) Multivariate bilinear time series: a stochastic alternative in population dynamics. Geophysical Research Abstracts, Volume 7, 02219 @Europeans Geosciences Union 2005.

Cathy, W. S. (1997) Detection of Additive Outliers in Bilinear Time Series. Computational Statistics and Data Analysis 24, 283-294

Doukhan, P., Latour, A., and Oraichi, D., (2006). A simple integer - valued bilinear time series model. Adv. Appl. Prob. 38: 559-578.

Drost F.C., van den Akker R., Werker, B.J.M. (2007). Note on integer - valued bilinear time series models. Econometrics and Finance group CentER, ISSN 09247815, Tilburg University, The Netherlands

Gonclaves, E., Jacob P. and Mendes-Lopes N. (2000) A decision procedure for bilinear time series based on the Asymptotic Separation. Statistics, 333-348

Granger, C. W. J. and Anderson, A. P. (1978) Introduction to Bilinear Time Series Models. Gottingen, Germany: Vandenhoeck and Ruprecht.

Haggan , V. and Oyetunji, O. B. (1980) On the Selection of Subset Autoregressive Time Series Models. UMIST Technical Report No. 124 (Dept. of Mathematics).

Krzanowski W.J. (1998) An Introduction to Statistical Modelling. Arnold, the UK.

Liu, J. (1992) On stationarity and Asymptotic Inference of Bilinear time series models. Statistica Sinica 2(2), 479494.

Ojo, J. F. (2009) The Estimation and Prediction of Autoregressive Moving Average Bilinear Time Series Models with Applications. Global Journal of Mathematics and Statistics Vol. 1, No2 Pages 111117.

Ojo, J. F. (2010) On the Estimation and Performance of One-Dimensional Autoregressive Integrated Moving Average Bilinear Time Series Models. Asian Journal of Mathematics and Statistics 3(4), 225-236. 
Pham, T.D. and Tran, L.T. (1981) On the First Order Bilinear Time Series Model. Jour. Appli. Prob. 18, 617627.

Rao, M. B., Rao, T. S and Walker, A. M. (1983) On the Existence of some Bilinear Time Series Models. Journal of Time Series Analysis 4(2), 60-76.

Shangodoyin, D. K. and Ojo, J. F (2003). On the Performance of Bilinear Time Series Autoregressive Moving Average Models. Journal of Nigerian Statistical Association 16, 1-12.

Shangodoyin, D. K., Ojo, J. F. And Kozak, M. (2010) Subsetting and Identification of

Optimal Models in One-Dimensional Bilinear Time Series Modelling. International
Journal of Management Science and Engineering Management 5(4), 252-260.

Gabr, M. M. and Subba Rao, T. (1981). The Estimation and Prediction of Subset Bilinear Time Series Models with Application. Journal of Time Series Analysis 2(3), 89100

Subba Rao, T. (1981) On theory of Bilinear time Series Models .Jour. R. Sta. Soc. B. 43, 244-255

Usoro, A. E. and Omekara C. O. (2008) Lowere Diagonal Bilinear Moving Average Vector Models. Advances in Applied Mathematical Analysis 3(1), 49-54

Wang H.B. and Wei B.C. (2004) Separable lower triangular bilinear model. J. Appl. Probab. 41(1), 221-235. 